Progress in Flight Physics 5 (2013) 601-620

DOI: 10.1051 /eucass/201305601

(C) Owned by the authors, published by EDP Sciences, 2013

\title{
IMPACT OF MULTIPOLE MATCHING RESOLUTION ON SUPERSONIC AIRCRAFT SONIC BOOM ASSESSMENT
}

\section{Salah El Din ${ }^{1}$, M.-C. Le Pape ${ }^{2}$, A. Minelli ${ }^{1}$, R. Grenon ${ }^{1}$, and G. Carrier ${ }^{1}$}

\author{
${ }^{1}$ Applied Aerodynamics Department, Civil Aircraft Unit \\ Office National d'Etudes et de Recherches Aérospatiales (ONERA) \\ 8 Rue des Vertugadins, Meudon 92190, France \\ ${ }^{2}$ Department of Numerical Simulation and Aeroacoustics, ONERA \\ BP 72-29, Av. de la Division Leclerc, Châtillon Cedex 92322, France
}

\begin{abstract}
Sonic boom assessment methods through numerical analysis have been ever-evolving since the development of the fundamental theory. With the growing need for efficient low-boom design tools and the increasing computational resources, original approaches using advanced numerical simulation techniques have been developed. Among the various existing methods, the three-layer resolution introduced in the 1990s is very convenient for low-boom aircraft design. It is based on near-field computational fluid dynamics (CFD) calculation which is matched with the far-field propagation code using a description of the source with multipoles before undergoing classical acoustic propagation through atmosphere. The present paper reviews the theory of the near- to far-field matching as well as the different resolution approaches and their limits. The impact of the resolution method on the ground propagated signal is also presented.
\end{abstract}

\section{NOMENCLATURE}

$A_{n} \quad$ multipole amplitude distribution

$C_{p} \quad$ pressure coefficient $\left(p-p_{\infty}\right) /\left(\gamma p_{\infty} \mathrm{M}_{\infty}^{2}\right)$

CFD Computational Fluid Dynamics

$F \quad$ Whitham $F$-function

$F_{n} \quad$ near-field (uncorrected) Fourier component over the pressure cylinder

$f_{t}(n)$ the $t$ th term in the expansion applied to the $n$th order multipole

$G_{n} \quad g_{n} / g_{\infty}$ 


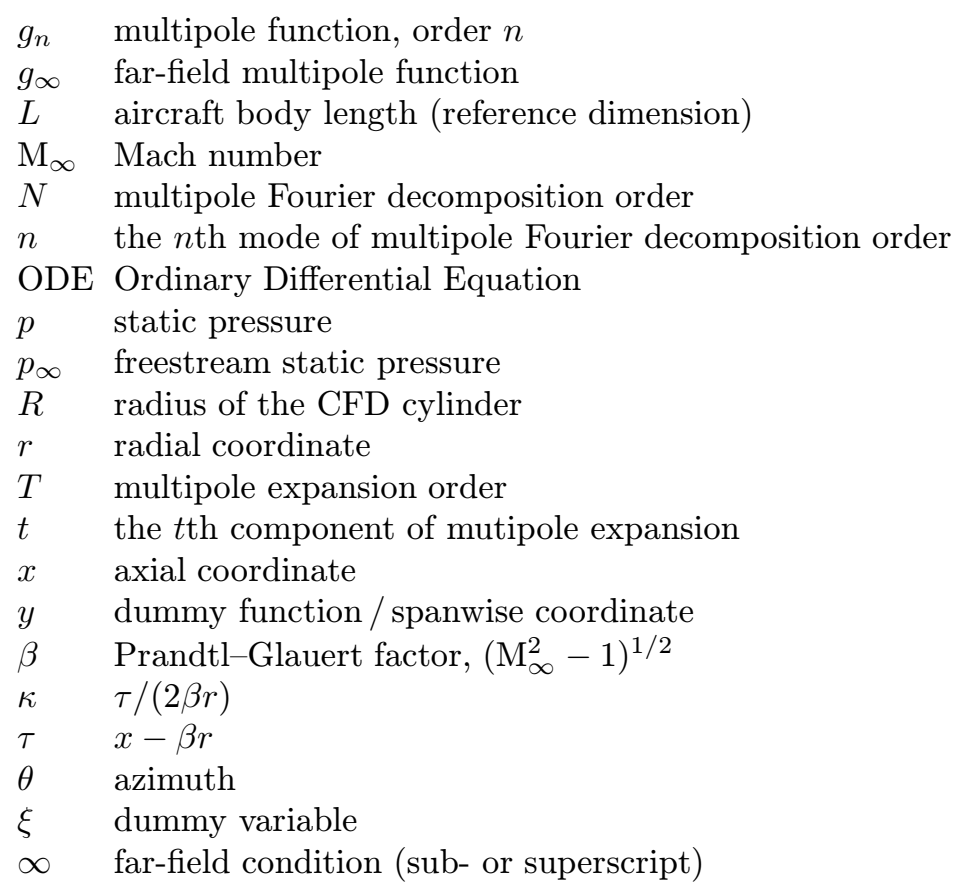

\section{INTRODUCTION}

Sonic boom prediction methodology has followed the evolution of the computational means since the first fundamental theoretical elements developed in the 1950s. The main challenge of sonic boom calculation is to be able to handle the wide range of scales at stake. Indeed, the pressure disturbances generated by the supersonic aircraft, which length $L$ is settled as the reference dimension, propagate through the atmosphere over hundreds of body lengths before reaching the ground form what is called sonic boom. In addition to the very different scales, the dominating physical phenomena change according to the distance to the source of the disturbances. Three regions have been identified by theory: the near-field, the mid-field, and the far-field. The near-field corresponds to a region within some body lengths, where the nonviscous aerodynamic small perturbation theory applies far from the boundary layer region. On the other hand, the propagation through the far-field, for tens of $L$ to the ground, is dominated by nonlinear acoustic phenomena. The near-field disturbances, before reaching the far-field, will undergo nonlinear distortion in a region where the corrected linear theory proposed by Whitham [1] and completed by Walkden for the winged body [2] is not valid any more. 
Most acknowledged accurate methods for sonic boom prediction are based on this three-layer decomposition. Plotkin and Page have published a synthetic and clear review [3] of the various existing methods used to assess sonic boom. Until the 1980s, the mid-field zone was not treated specifically and efforts were made to improve the accuracy of the near-field three-dimensional (3D) CFD computations. The far-field propagation was performed using nonlinear acoustic codes following ray tracing technique. Thomas published an algorithm to compute sonic boom using the waveform parameter method [4] as a new approach compared to the work of Hayes et al. [5] who used the age parameter and the area balancing rules. Darden also proposed a modified method of characteristics (MMOC) to solve propagation from near-field to the ground in the symmetry plane taking into account cross flow effects [6].

Since the 1990s, the increasing use of automated numerical exploration tools for low boom aircraft design called for a more accurate and efficient sonic boom evaluation in terms of computation time reduction and prediction accuracy and robustness. Even though attempts to perform CFD from the body to the ground with both evolutive schemes and equation formulations, as proposed by Kandil et al. [7] or hybrid approaches more recently developed by Alauzet and Loseille performing calculations up to the far-field before ray tracing propagation [8], the three-layer approach remains very competitive. Page and Plotkin proposed an efficient three-layer approach [9] based on CFD computations for the near-field, a near- to far-field matching through multipole decomposition of the source of disturbances and an acoustic propagation through atmosphere. The matching approach exploits the work of George [10] on sonic boom minimization. The near- to far-field matching which is presented in this paper is based on this multipole matching method. The numerical resolution is described as developed at ONERA following the work of I. Salah El Din [11] as well as the modification of the algorithm introduced by Rallabhandi and Mavris [12].

\section{SONIC BOOM THREE-LAYER APPROACH}

\section{Three-Layer Approach Principle}

As mentioned before, the three-layer approach is consistent with the undergoing evolution of the physics through the whole disturbances propagation from the source to the ground. In the vicinity of the supersonic aircraft, the aerodynamic phenomena dominate. In this region, the aerodynamic flow is governed by the nonlinear Navier-Stokes equations and includes 3D features with scales proportional to the aircraft length. However, considering the current knowledge and computational means, using a nonviscous CFD solver is very convenient to predict the near-field pressure disturbances "far" from the viscous boundary 
layer region. The nonlinear 3D aeroacoustic distortion due to the propagation through the mid-field is assessed using the matching method proposed by Page and Plotkin [9]. Finally, the long distance acoustic propagation through standard stratified atmosphere is carried out with a specific acoustic code.

The ONERA sonic boom prediction methodology is illustrated in Fig. 1. Layer 1 corresponds to the near-field aerodynamic flow prediction with CFD. Layer 3 is the atmospheric propagation of the sonic boom with an acoustic code, while layer 2 ensures a natural matching between the near-field aerodynamic data and the native inputs of the acoustic code. A limit of this approach is that it does not provide the mid-field flow and it is assumed that acoustic and aerodynamic effects between the near- and the far-field can be computed separately and added to each other. Some applications of the methodology are presented in [13].

\section{Layer 1: Near-Field Aerodynamic Calculation by Computational Fluid Dynamics}

For a given aircraft geometry, typically a wing-body glider type configuration in our case, a structured mesh is generated using an automated ONERA in-house tool. The ONERA elsA [14] CFD software is used to perform the nonviscous calculation solving the 3D steady Euler equations of the supersonic flow. The computational domain can reasonably reach few body lengths and the automatic mesh adaptation processes around the shock waves allow an accurate near-field evaluation at this distance.

\section{Layer 2: Multipole Matching Method}

The CFD calculation provides the aerodynamic pressure in the near-field. As an input to the matching method, the pressure is extracted on a cylinder around the aircraft aligned with the flow direction. The matching allows making the pressure signature compliant with the acoustical assumption of local axisymmetry field which is necessary to nonlinear acoustic propagation theory and ensured with an adequate choice of $r / L$. The pressure coefficient, corresponding to the nondimensioned pressure fluctuation with respect to the freestream conditions at a distance $r$ from the source, on the azimuth $\theta$, and at streamwise position $\tau$ is directly related to the Witham function $F$, as given below:

$$
C_{p}(\tau, \theta, r)=\frac{p-p_{\infty}}{\gamma p_{\infty} \mathrm{M}_{\infty}^{2} / 2}=\sqrt{\frac{2}{\beta r}} F(\tau, \theta, r) .
$$

George [10] proposed to model the aircraft as a distribution of multipoles generating equivalent near-field disturbances. Page and Plotkin [9] extended 


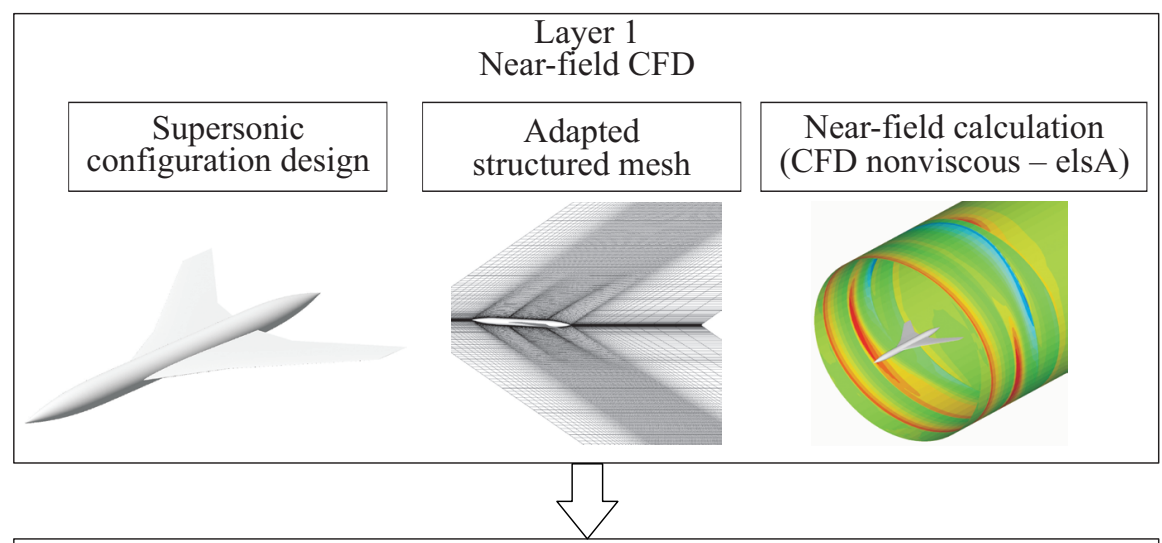

Layer 2

Multipole matching

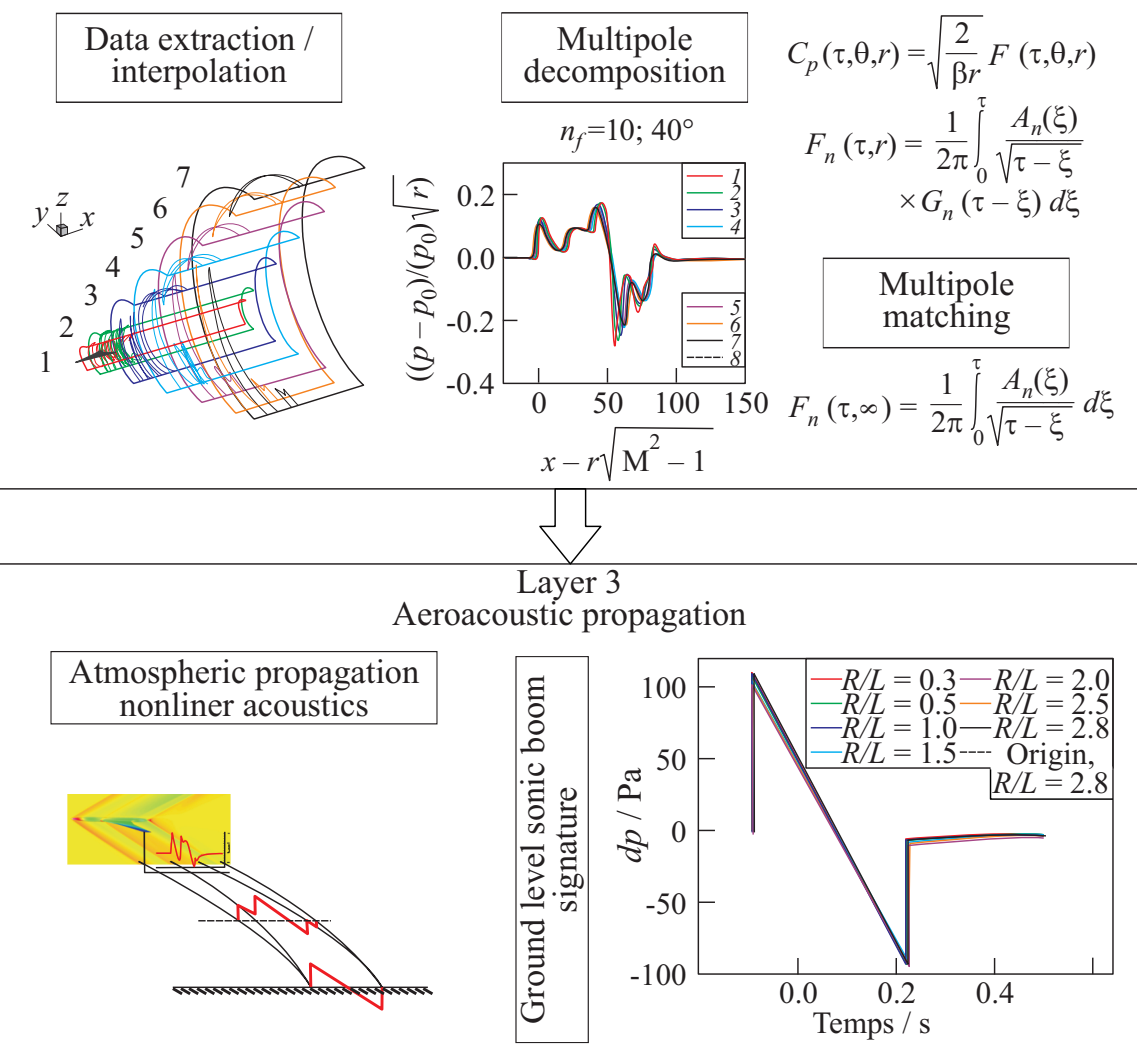

Figure 1 Three-layer sonic boom prediction approach. 
the formulation to the far-field sources as a near-field data correction taking into account the azimuthal distortion occurring during the propagation from the near- to the far-field. The matching method has been implemented in an ONERA in-house code following the work of Salah El Din [11].

The first step in the matching method is to decompose the Whitham function, following Fourier type decomposition, into $F_{n}$ components depending on the distance from the source and the length of the signal which are related to the pressure coefficient $C p$, as indicated below:

$$
\begin{aligned}
F(\tau, r, \theta) & =\sum_{n=0}^{\infty} F_{n}^{\infty}(\tau, r) \cos (n \theta) ; \\
F_{0}(\tau, r) & =\sqrt{\frac{\beta r}{2}} \frac{1}{\pi} \int_{0}^{\pi} C_{p}(\tau, r, \theta) d \theta ; \\
F_{n}(\tau, r) & =\sqrt{\frac{\beta r}{2}} \frac{2}{\pi} \int_{0}^{\pi} C_{p}(\tau, r, \theta) \cos (n \theta) d \theta .
\end{aligned}
$$

Modeling the source as a distribution of multipoles as a general solution of the perturbation potential equation, as proposed by George, leads to an expression of $g_{n}$ multipole function family with an amplitude $A_{n}$. The $G_{n}$ function is introduced as the ratio between the $n$th order multipole function with the farfield multipole function $g_{\infty}$ obtained by setting $r$ to $+\infty$ :

$$
F_{n}(\tau, r)=\frac{1}{2 \pi} \int_{0}^{\pi} \frac{A_{n}(\xi)}{\sqrt{\tau-\xi}} G_{n}(\tau-\xi, r) d \xi
$$

with

$$
\left.\begin{array}{rl}
G_{n}(\tau, r) & =\frac{g_{n}(\tau, r)}{g_{\infty}(\tau, r)} \\
g_{n}(\tau, r) & =-\frac{1}{2 \pi} \frac{\cosh (n \arg \cosh (1+\tau /(\beta r)))}{\sqrt{\tau /(2 \beta r)} \sqrt{(1+\tau /(2 \beta r))}} ; \\
g_{\infty}(\tau, r) & =-\frac{1}{2 \pi} \frac{1}{\sqrt{\tau /(2 \beta r)}} .
\end{array}\right\}
$$

The far-field matched function results in suppressing the dependency on the distance from the source $r$ of the $F_{n}$ components. The matched Whitham function is reassembled using the matched components: 


$$
\begin{aligned}
& F_{n}(\tau, \infty)=\frac{1}{2 \pi} \int_{0}^{\pi} \frac{A_{n}(\xi)}{\sqrt{\tau-\xi}} d \xi \\
& F_{\infty}(\tau, \theta)=\sum_{n=0}^{\infty} F_{n}^{\infty}(\tau, \infty) \cos (n \theta) .
\end{aligned}
$$

The resulting matched Whitham function can, therefore, be used for nonlinear ray tracing acoustic propagation.

\section{Layer 3: Atmospheric NonlinearAacoustic Propagation Method}

The last step of the pressure disturbances propagation toward the ground is the acoustic propagation of the matched signal using the acoustic propagation code, TRAPS [15]. TRAPS is a dedicated sonic boom propagation code based on the inviscid nonlinear acoustic theory. It uses a ray-tracing approach to account for the refraction phenomena occurring during the propagation through a stratified atmosphere with vertical temperature, cross wind, and density gradients, to evaluate the extent of the "primary carpet," i.e., the width of the corridor underneath the aircraft trajectory directly affected by sonic boom. Along each acoustic ray, the Whitham theory (first-order correction to the linear supersonic theory) is used to predict the evolution of the shape of the sonic boom pressure signal.

\section{MATCHING METHOD EQUATIONS RESOLUTION}

\subsection{Direct Resolution Approach}

What is called direct resolution here consists in finding explicitly the $A_{n}$ amplitude functions used for the far-field Whitham function reconstruction (see Eq. (5)). The main difficulty in the multipole matching method lies in the formulation of the near-field $F_{n}$ functions in which small amplitude levels of $A_{n}$ are combined with high levels of the multipole functions $G_{n}$ near the source of disturbances. Figure 2 illustrates a typical evolution of the $G_{n}$ functions with respect to the nondimensioned distance to the source $r / L$ for $n$ in $\{0,1, \ldots, 5\}$. As the order rises, the values reached by $G_{n}$ get higher at a given $r / L$. The capability of increasing the order will allow matching flow fields of increasing complexity.

The $A_{n}$ functions can be obtained by identification of the two formulations of the $F_{n}(\tau, r)$ found by combination of Eqs. (3) to (6) and compute the corresponding $F_{n}(\tau, \infty)$. Two approaches have been tested: the first one by assuming 


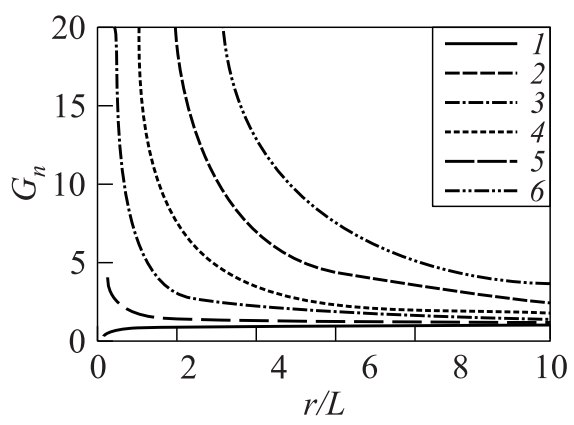

Figure 2 Typical $G_{n}$ function distribution for $n=0(1) ; 1(2) ; 2(3) ; 3$ (4); 4 (5); and $5(6)$

that the $A_{n}$ are constant by part on the subintervals $\left[\tau_{k}, \tau_{k+1}\right]$ of the streamwise discretization ranging within $[0, \tau]$; the second attempt consisted in rising the order of the $A_{n}$ discretization to linear by part evolutions with continuity ensured at the subdiscretization points $\tau_{k}$ :

$$
\begin{aligned}
& A_{n}(\tau)=a_{k} \quad \forall \tau \in\left[\tau_{k} ; \tau_{k+1}\right], \\
& F_{n}\left(\tau_{p}, \infty\right)=\frac{1}{2 \pi} \int_{0}^{\tau_{p}} \frac{A_{n}(t)}{\sqrt{\tau_{p}-t}} d t=\frac{1}{2 \pi} \sum_{k=0}^{p-1} \int_{\tau_{k}}^{\tau_{k+1}} \frac{a_{k}}{\sqrt{\tau_{p}-t}} d t ; \\
& A_{n}(\tau)=a_{k} \tau+b_{k} \quad \forall \tau \in\left[\tau_{k} ; \tau_{k+1}\right], \\
& F_{n}\left(\tau_{p}, \infty\right)=\frac{1}{2 \pi} \int_{0}^{\tau_{p}} \frac{A_{n}(t)}{\sqrt{\tau_{p}-t}} d t=\frac{1}{2 \pi} \sum_{k=0}^{p-1} \int_{\tau_{k}}^{\tau_{k+1}} \frac{a_{k} t+b_{k}}{\sqrt{\tau_{p}-t}} d t .
\end{aligned}
$$

The system to be solved when $A_{n}$ is modeled with a function linear by part is given below:

$$
\begin{aligned}
& A_{n}(\tau)=a_{k} \tau+b_{k} \forall \tau \in\left[\tau_{k} ; \tau_{k+1}\right] \\
& F_{n}\left(\tau_{1}, r\right)=\frac{a_{0}}{2 \pi} \int_{\tau_{0}}^{\tau_{1}} \frac{t}{\sqrt{\tau_{1}-t}} G_{n}\left(\tau_{1}-t, r\right) d t+\frac{b_{0}}{2 \pi} \int_{\tau_{0}}^{\tau_{1}} \frac{1}{\sqrt{\tau_{1}-t}} G_{n}\left(\tau_{1}-t, r\right) d t
\end{aligned}
$$




$$
\begin{gathered}
a_{p}=\frac{2 \pi F_{n}\left(\tau_{p+1}, r\right)-A_{n}\left(\tau_{p}\right) \int_{\tau_{p}}^{\tau_{p+1}}\left(1 / \sqrt{\tau_{p+1}-t}\right) G_{n}\left(\tau_{p+1}-t, r\right) d t}{\int_{\tau_{p}}^{\tau_{p+1}}\left(t-\tau_{p}\right) / \sqrt{t_{p+1}-t} G_{n}\left(\tau_{p+1}-t, r\right) d t} \\
-\frac{\sum_{k=0}^{p-1} \int_{\tau_{k}}^{\tau_{k+1}}\left(a_{k} t+b_{k}\right) / \sqrt{\tau_{p+1}-t} G_{n}\left(\tau_{p+1}-t, r\right) d t}{\int_{\tau_{p}}^{\tau_{p+1}}\left(t-\tau_{p}\right) / \sqrt{\tau_{p+1}-t} G_{n}\left(\tau_{p+1}-t, r\right) d t} ;
\end{gathered}
$$$$
b_{p}=A_{n}\left(\tau_{p}\right)-a_{p} \tau_{p}
$$

with

$$
\begin{aligned}
A_{n}\left(\tau_{p}\right) & =a_{p-1} \tau_{p}+b_{p-1} \\
F_{n}\left(\tau_{p+1}, r\right) & =\sqrt{\frac{\beta R}{2}} \frac{2}{\pi} \int_{0}^{\pi} C_{p}\left(\tau_{p+1}, \theta, r\right) \cos (n \theta) d \theta .
\end{aligned}
$$

Each component can be computed separately using integration by parts.

\subsection{Differential Resolution Approach}

The second approach for the multipole matching system resolution has been proposed by Rallabhandi and Mavris [12]. The main idea is to reformulate the problem through multiple derivations to change the integral inversion problem into a classical ODE system. The first step to find the equivalent ODE problem consists in the expansion of the $G_{n}$ functions around $\kappa=\tau /(2 \beta r)$. The compact formulation of the $T$ th order expansion is given below:

$$
G_{n}(\tau, r)=1+\sum_{t=0}^{T-1} f_{t}(n)\left(\frac{\tau}{\beta r}\right)^{i+1}+O\left(\tau^{T+1}\right) .
$$

The $f_{t}$ functions are the polynomial functions of order $2(t+1)$. As samples, $f_{0}, f_{1}$, and $f_{5}$ are given in the following equation set:

$$
f_{0}(n)=n^{2}-\frac{1}{4} ; \quad f_{1}(n)=\frac{16 n^{4}-40 n^{2}+9}{96} ; \cdots ;
$$




$$
\begin{aligned}
f_{5}(n)=\frac{1}{64}\left[\frac{231}{1024}-\frac{7156487}{6652800} n^{2}\right. & +\frac{1997021}{2721600} n^{4}-\frac{28067}{170100} n^{6} \\
& \left.+\frac{871}{56700} n^{8}-\frac{26}{42525} n^{10}+\frac{4}{467775} n^{12}\right] ; \cdots
\end{aligned}
$$

One of the limitations of the method is that the function coefficients can reach values which cannot be handled by the computations capabilities.

The approximation error is shown in Fig. 3 with respect to $x / L$ and $r / L$. The expansion of the $G_{n}$ function leads to approximations which are significant as it gets close to the source located at $r / L=0$. As the multipole order considered gets higher, the error propagates upstream (from $x / L=1$ toward 0 ). The impact of the error on the matched signal may not be critical even at small $r / L$ (typically smaller than 1) as the pole contribution, depending on its amplitude, may be negligible.

By introducing the $G_{n}$ formulation in Eq. (3) and by performing incremental multiple integrations by part, the $n$th component of the Whitham function can be expressed as follows:

$$
F_{n}(\tau, r)=F_{n}(\tau, \infty)+\sum_{t=0}^{T-1} \frac{f_{t}(n)}{(\beta r)^{t+1}}(t+1) ! \int_{0}^{\tau} \cdots \int_{0}^{\xi_{t}} F_{n}\left(\xi_{t}, \infty\right) d \xi_{t} \cdots d \xi_{1}
$$

Introducing a dummy function $y$, Eq. (7) can be written as the sum of the derivation of $y$ up to the $T$ th order:

$$
\begin{gathered}
y(\tau)=\int_{0}^{\tau} \cdots \int_{0}^{\xi_{T}} F_{n}\left(\xi_{T}, \infty\right) d \xi_{T} \cdots d \xi_{1} \\
F_{n}(\tau, r)=y^{(T)}+\sum_{t=0}^{T-1} \frac{f_{t}(n)}{(\beta r)^{t+1}}(t+1) ! y^{(T-1-t)} .
\end{gathered}
$$

By setting the boundary condition equal to 0 as initial value, the problem has been shifted to the resolution of an ODE which can be split into a $T$ first-order differential equation system.

\section{APPLICATION CASE}

\subsection{Test Case Geometry, Mesh, and Computational Fluid Dynamics Specifications}

In order to test the impact of the various resolution methods, a glider test case has been defined. To generate a complex $3 \mathrm{D}$ disturbance field with multiple 

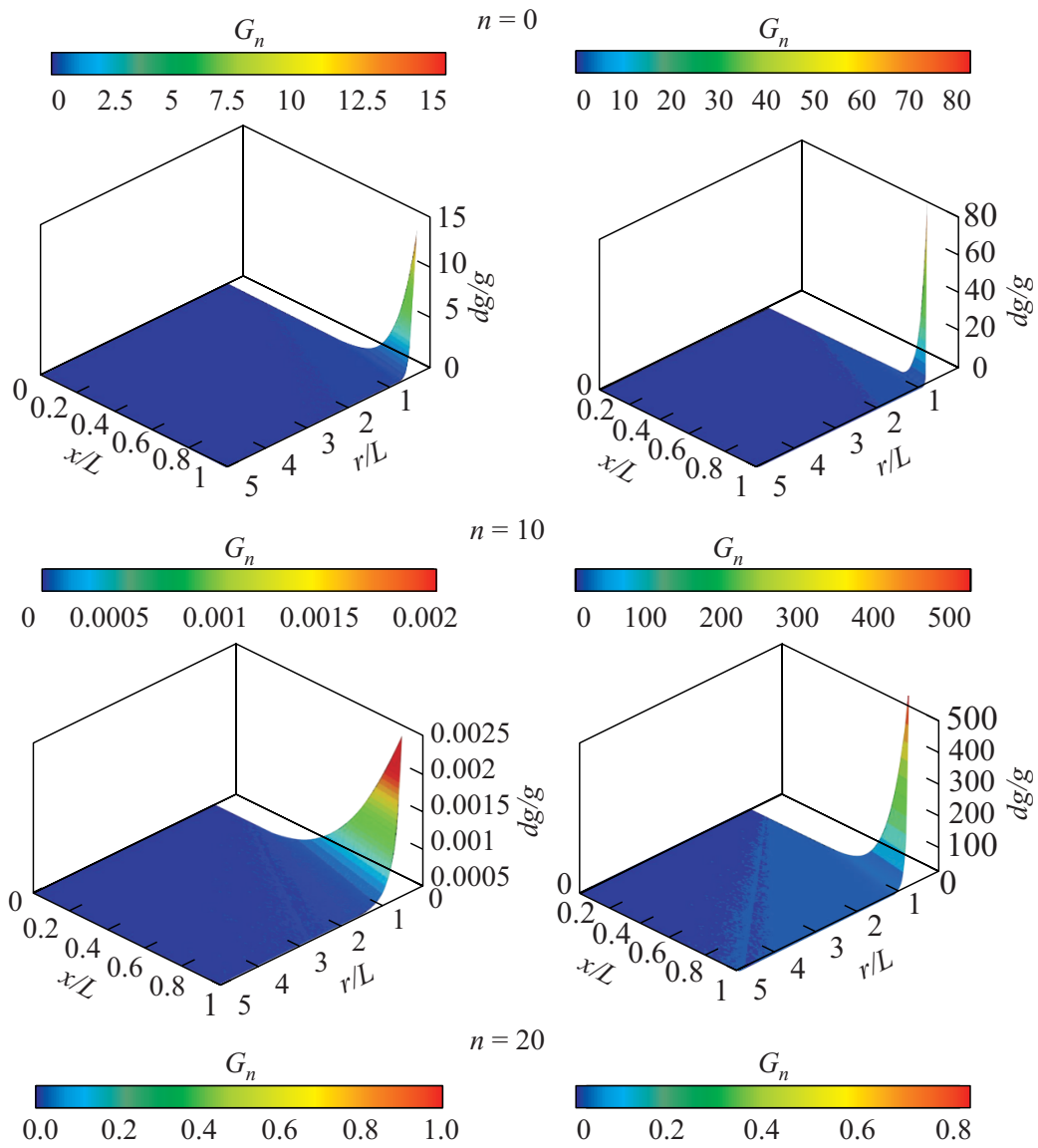

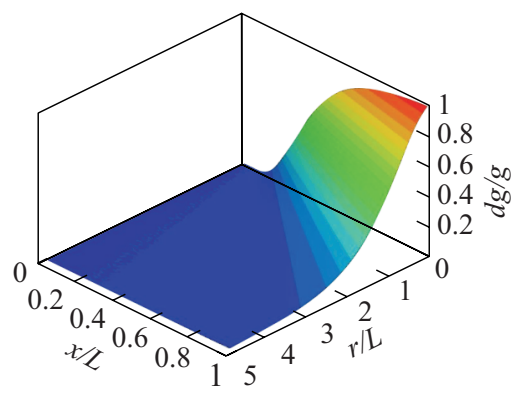

(a)

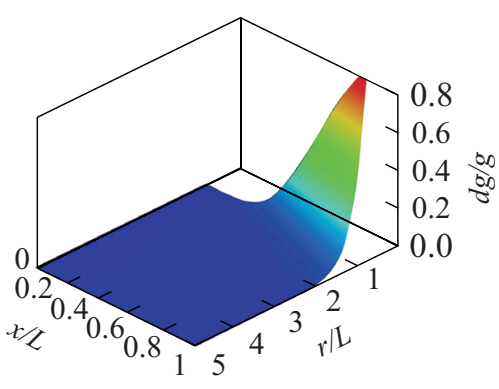

(b)

Figure 3 Error on $G_{n}$ due to analytical expansion formulation: $(a) T=10$; and (b) $T=16$. 

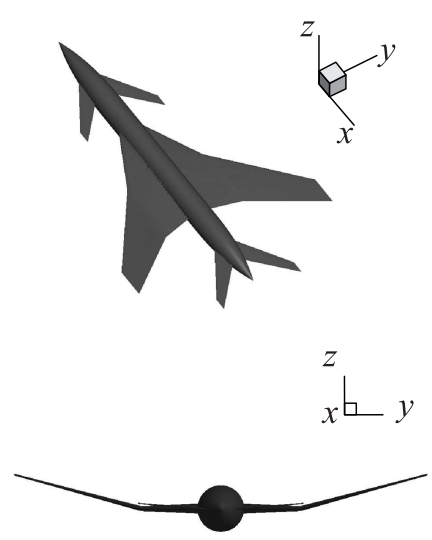

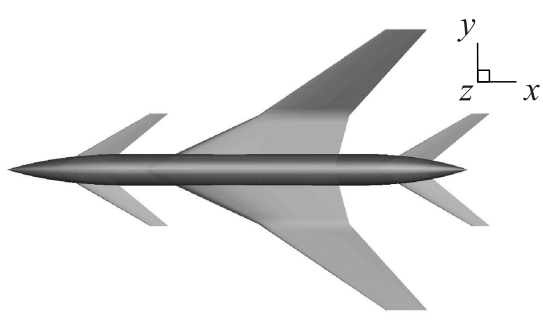

$z$

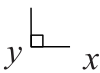

Figure 4 Test case geometry

shock wave patterns, nose and tail lifting surfaces have been added and dihedral angles applied to each element. The resulting geometry is illustrated in Fig. 4.

An ONERA in-house mesh generation code generates a structured H-type mesh around the chosen configuration, with mesh refinement in regions where strong shock waves are expected to appear according to supersonic theory as shown in Fig. 5. The computation domain is extended up to four times of the body length.

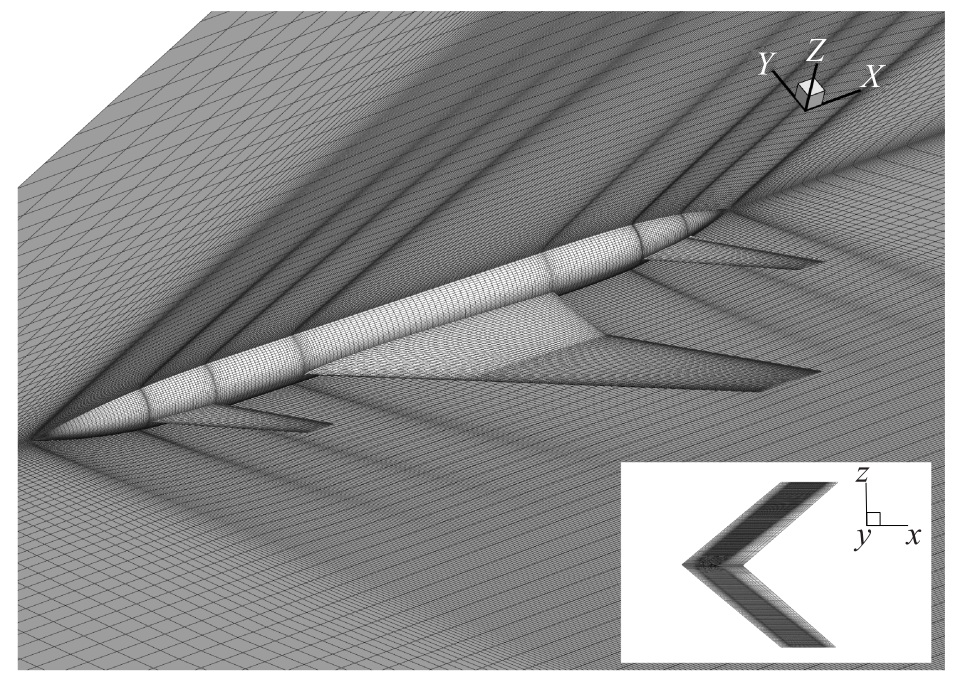

Figure 5 Test case adapted structured mesh 
The nonviscous computations are performed using elsA [14] solver at the following flight conditions: flight Mach number $\mathrm{M}=1.6$ and angle of attack of the glider configuration $=2.35^{\circ}$. The Euler equations are solved using a Roe scheme with Van Albada limiter.

\subsection{Near-Field Data Analysis}

The converged computation provides the state variables in the flow around the aircraft. The observation of slices normal to the freestream axis given in Fig. 6 confirms the complex 3D patterns of the aerodynamic field disturbances.
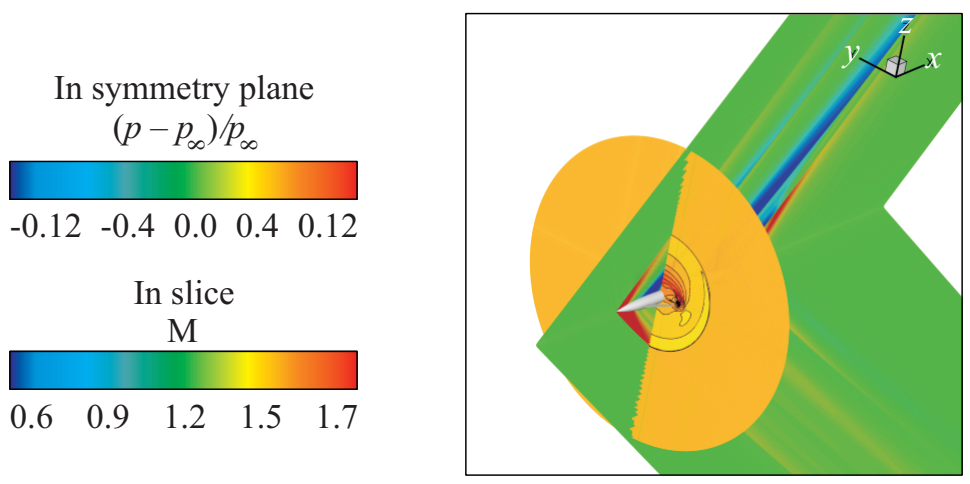

(a)

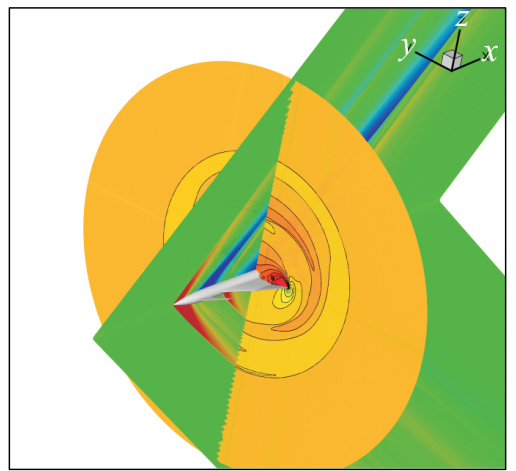

(b)

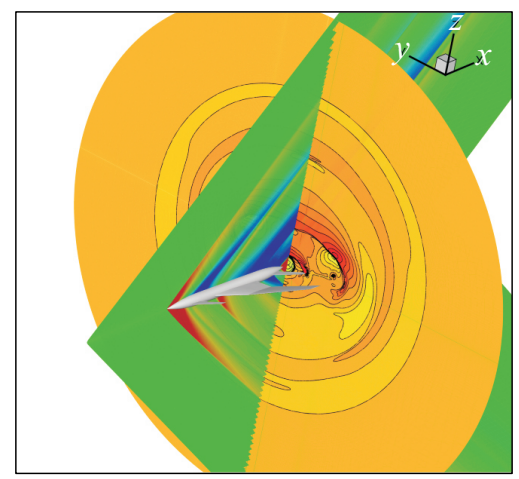

(c)

Figure 6 The CFD volume field pattern of pressure in symmetry plane and iso-Mach contours in flow slices, respectively, at $x / L=0.3(a) ; 0.6(b)$; and $1.3(c)$. 


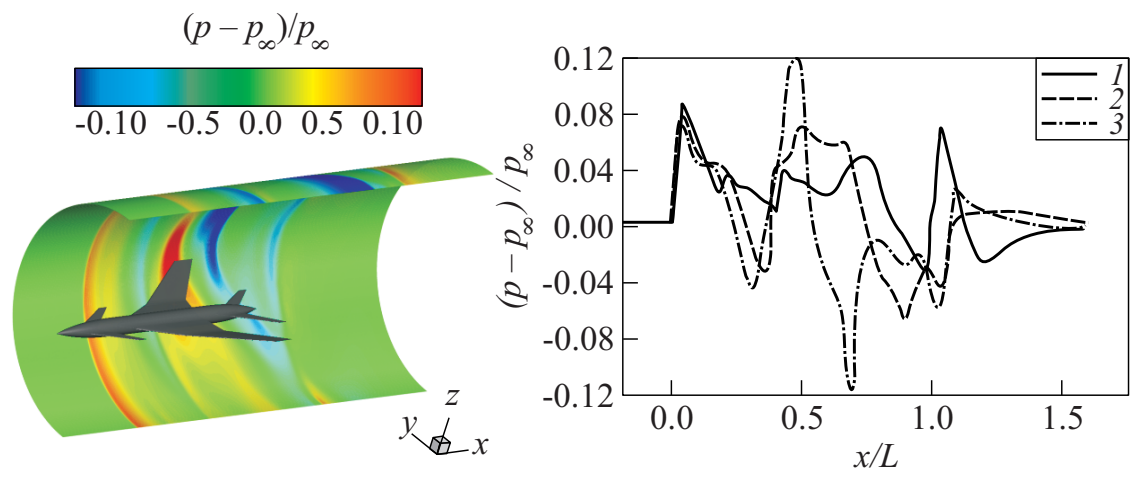

Figure 7 Cylinder pressure disturbances data extraction at $r / L=0.5: 1-\theta=0^{\circ}$; $2-54^{\circ}$; and $3-\theta=90^{\circ}$.

The static pressure on a cylinder is interpolated from the flow field at $r / L$ $=0.5$. The resulting pressure field and extraction lines at three azimuthal angles are provided in Fig. 7: the flow undergoes a sequence of multiple shocks, progressive compressions, and expansions of varying amplitude. The $3 \mathrm{D}$ behavior of the flow is illustrated again with a strongly varying wing shock wave reaching a peak level for $\theta=90^{\circ}$.

\subsection{Near- to Far-Field Matching}

The different matching resolution methods have been applied to the previously described near-field data. The conclusions drawn from the analysis of the present data give indications about the behavior of the matching method resolution, but should be used on different near-field data sets with caution. Indeed, it has been observed that convergence can be case dependent as the various decompositions are explicitly related to the pressure signature.

Both methods, as presented in Fig. 8, lead to converged signals at a decomposition order of $n=10$. Notice that the reference signal has undergone significant modifications through the matching process. The only unmodified part of the signal is the nose shock and the following expansion, which can easily be explained by the fact that the azimuthal evolution of the disturbances around the nose shock is very small. The differential approach diverged for a multipolar decomposition higher than $n=17$ for an expansion order of $T=14$.

Once the convergence ensured, with respect to $\mathrm{n}$ for both methods, the matched signals have been compared (Fig. 9) at three representative azimuths: $\theta=0^{\circ}$ (under track), $54^{\circ}$ (first completely refracted ray azimuth angle, limiting the primary carpet), and $90^{\circ}$ (maximum of wing shock). 


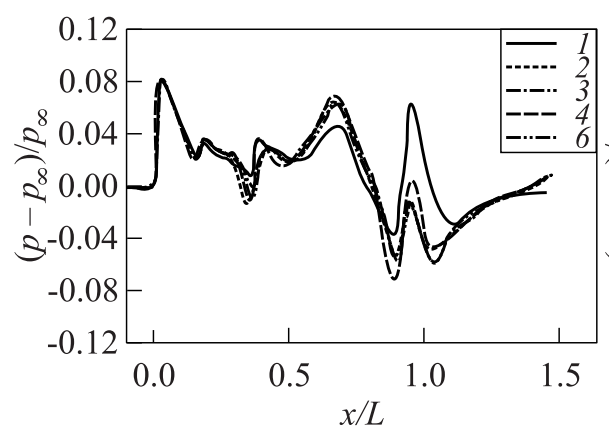

(a)

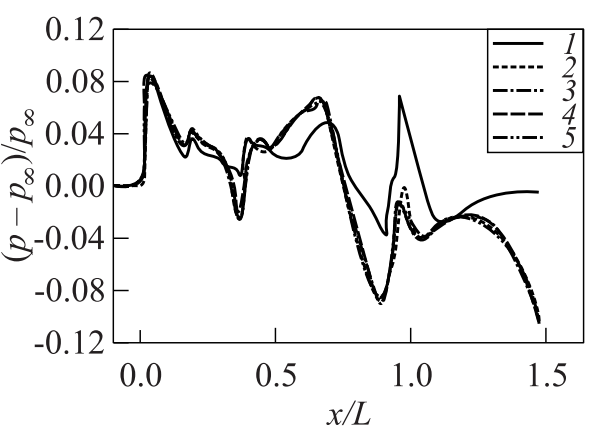

(b)

Figure 8 Sample convergence curves of pressure disturbances with respect to multipole decomposition order at $r / L=0.5, \theta=0^{\circ}, \mathrm{M}=1.6$ by direct $(a)$ and differential $(T=14)(b)$ resolutions: 1 - reference unmatched; $2-n=5 ; 3-10 ; 4-15 ; 5-$ 17 ; and $6-n=20$

Just as for the $\theta=0^{\circ}$ signature, the nose shock/expansion remains unchanged by the matching at $\theta=54^{\circ}$ as well as at $\theta=90^{\circ}$. The main distortions which can be observed appear at the wing shock contribution and impact the whole signal downstream. The rear part of the signal, whatever the method, does not converge toward the undisturbed freestream state as it should. This is due to the numerical errors and instabilities of the system resolution as very high order expansion are considered as well as a high expansion order compared to the one usually found in the literature (see $[9,12]$ ). Furthermore, as the data extraction distance of $r / L=0.5$ is small if compared to the commonly used one $(r / L$ $>1$ ), the matching must face stronger local gradients. Both methods show that the matching technique shifts the global energy signature toward the symmetry plane: the compression/expansion wave amplitudes decrease for $\theta=90^{\circ}$ whereas they increase for $\theta=0^{\circ}$ and $54^{\circ}$.

The limit of the numerical resolution is illustrated in Figs. 10 and 11. For the chosen configuration, the periodic instabilities appear in both cases but for different reasons. Concerning the direct approach, oscillations appear downstream and propagate upstream beyond a critical $n=22$. Figure 10 shows that at $n=24$, the oscillations begin to reach the "useful" part of the signature. Such behavior is consistent with the evolution of the $G_{n}$ functions illustrated in Fig. 2 as they reach very high values when $n$ increases. On the other hand, concerning the differentiation approach, the divergence appears for smaller values of multipole order. This is explained by the analysis of Fig. 3 as the error made on $G_{n}$ approximation by the expanded formulation (6) increases with the order of the multipole. Notice that the numerical oscillatory phenomenon observed in Fig. 11 impact every multipole decompo- 


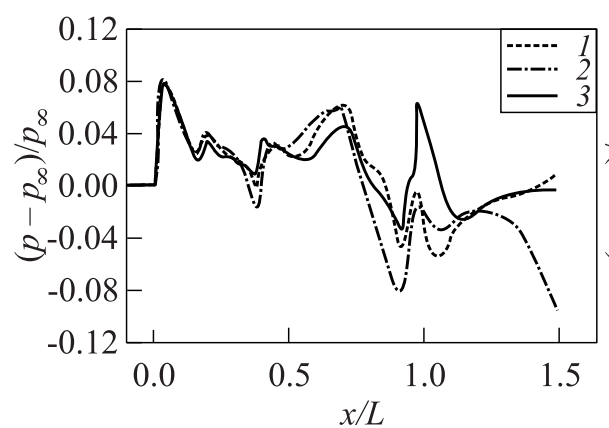

(a)

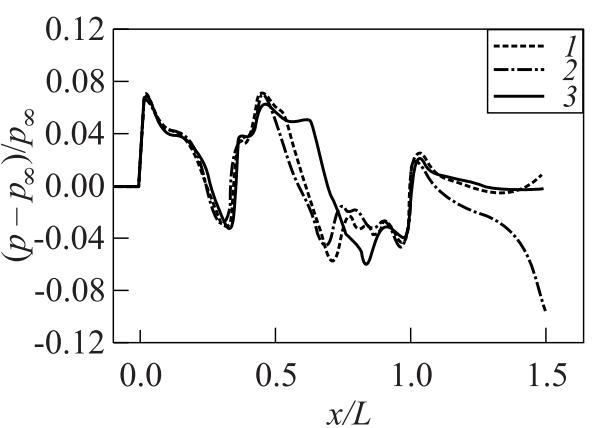

(b)

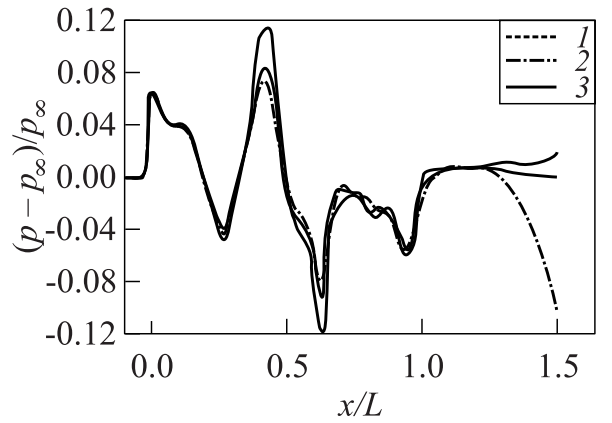

(c)

Figure 9 Comparison of the matched pressure disturbances curves between the direct (1) and differential (2) resolution methods at $r / L=0.5, \mathrm{M}=1.6$, and $\theta=0^{\circ}(a)$; $54^{\circ}(b)$; and $90^{\circ}(c): 1-n=20 ; 2-n=17, T=14 ;$ and $3-\left(p-p_{\infty}\right) / p_{\infty}$

sition order and it has a lower frequency than the one using the direct approach.

\subsection{Acoustic Propagation}

The undertrack signal has been propagated using the TRAPS code from the flight altitude to the ground level as shown in Fig. 12. Indeed, the region most impacted by the matching is located around the symmetry plane. The propagation of the uncorrected reference pressure leads to a multiple shocks patterned ground signature. The signal correction due to the matching has a significant impact on the resulting ground signature as it increases its length as well as the shock amplitude and all the shocks have coalesced. The effect is consistently reproduced with both matching resolution methods, the direct resolution leading to a longer ground signature. Notice that the decomposition and expansion 


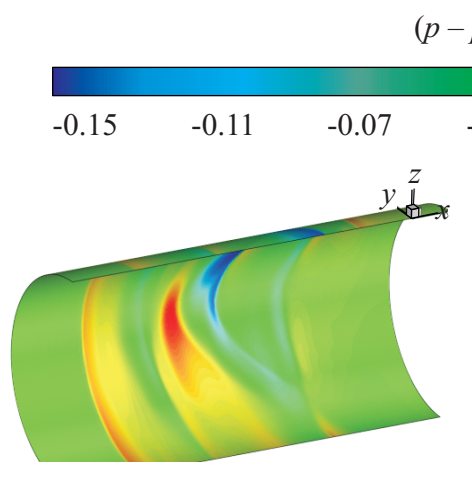

(a)

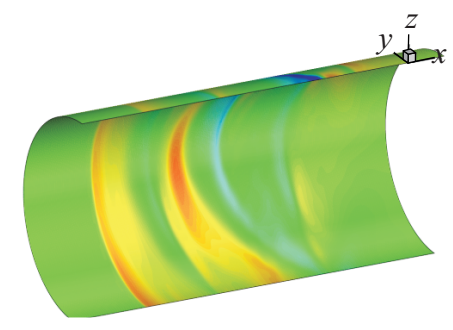

(c) $\left(p-p_{\infty}\right) / p_{\infty}$

$\begin{array}{llll}-0.03 & 0.01 & 0.05 & 0.09\end{array}$

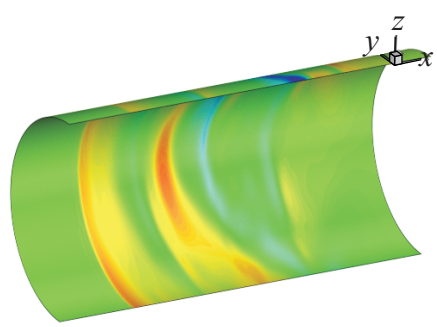

(b)

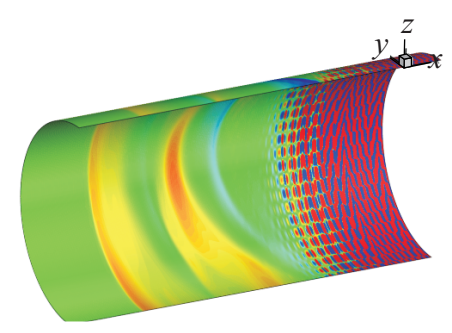

(d)

Figure 10 Divergence of direct resolution method: $(a)$ reference unmatched; $(b) n$ $=5 ;(c) 10 ;$ and $(d) n=24$.

orders have been chosen to avoid the oscillations. The ground signature obtained with the unmatched CFD near-field is nonsymmetric, which is an indication that $r / L$ is too small. This undesirable feature is corrected by the matching with both methods, enabling to keep the extraction near the aircraft.

\section{CONCLUDING REMARKS}

In the process of sonic boom assessment, the multipole matching method is considered as an efficient way to take into account near- to far-field nonlinear effects. Its integration in a three-layer resolution approach definitely improves the accuracy of the ground pressure signature and allows the extraction of the CFD data closer to the aircraft. It appears as a good compromise, an alternative solution between limited analytical resolution methods like MMOC and explicit costly CFD from body to ground approaches. 


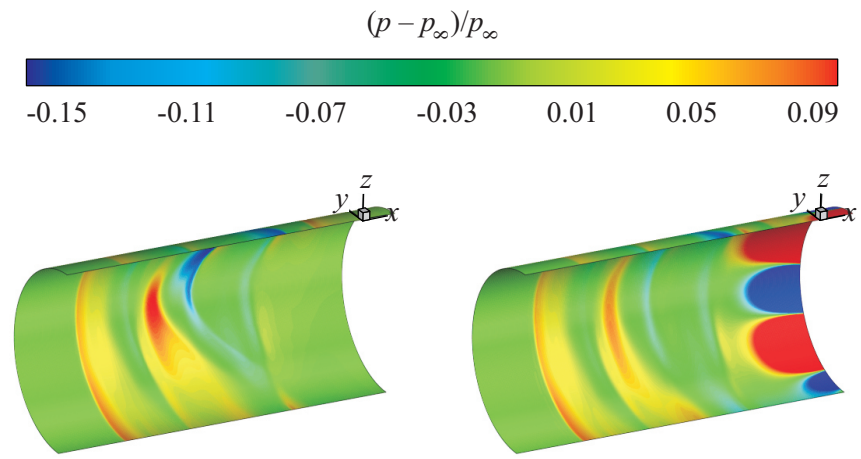

(a)

(b)

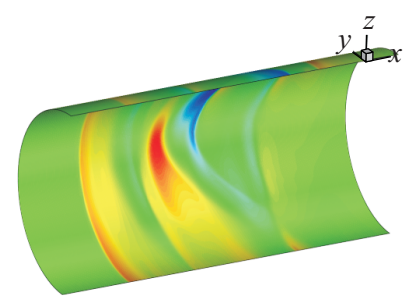

(c)

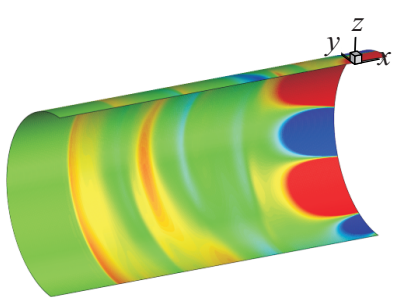

(d)

Figure 11 Divergence of differential resolution method $(T=16)$ : $(a)$ reference unmatched; $(b) n=5 ;(c) 10 ;$ and $(d) n=17$.

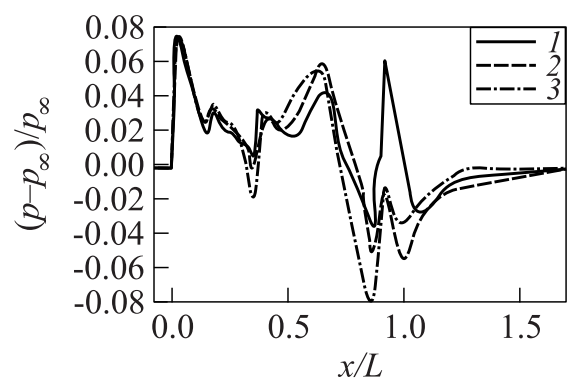

(a)

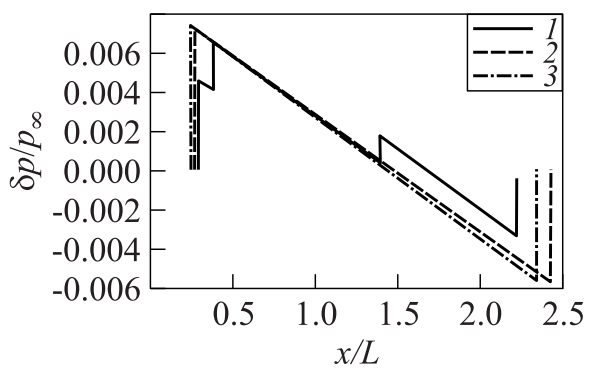

(b)

Figure 12 Impact of matching multipole resolution method at $r / L=0.5$ on nearfield $(a)$ and ground $(b)$ signatures, $\theta=0^{\circ}: 1-$ reference unmatched; 2 - direct resolution, $n=15$; and $3-\mathrm{ODE}, n=15, T=12$ 
The mathematical formulation of the problem has been described as well as the different resolution approaches, named "direct" and "differential" multipole matching. Both methods are mathematically valid and the sensitivity of both methods to the key parameters, which are the number of poles used to describe the source of pressure disturbances and the multipole functions expansion order, have been presented. An application case has been defined and the near-field analysis performed. Both matching resolution approaches have been applied to this test case and their behavior as well as the impact of the matching on the reconstructed pressure field distribution have been analyzed in detail. Finally, both methods have been pushed to their limits in terms of numerical stability.

The impact of the near- to far-field matching on the ground level sonic boom has been evaluated. Considering that the near-field data have been extracted very close to the source compared to that is presented in the literature [8], a study of convergence in $r / L$ should be performed to validate the pressure levels. The problems of numerical resolution of both matching methods on the downstream part of the matched signal must also be tackled to improve the robustness and the accuracy of the global sonic boom prediction process. However, the whole sonic boom evaluation process is operational [13] and has shown its efficiency for various application geometries and flight conditions in international projects such as HISAC [16].

\section{REFERENCES}

1. Whitham, G. B. 1952. The flow pattern of a supersonic projectile. Communications Pure Appl. Math. 5:301-48.

2. Walkden, F. 1958. The shock pattern of a wing-body combination, far from the flight path. The Aeronautical Quarterly IX:164-94.

3. Plotkin, K., and J. Page. 2002. Extrapolation of sonic boom signatures from CFD solution. AIAA Paper No. 2002-0922.

4. Thomas, C. L. 1972. Extrapolation of sonic boom pressure signatures by the waveform parameter method. NASA Technical, TN D-6832.

5. Hayes, W. C., R. C. Haefeli, and H. E. Kulsrud. 1969. Sonic boom propagation in a stratified atmosphere, with computer program. NASA CR-1299.

6. Darden, C. M. 1984. An analysis of shock coalescence including three-dimensional effects with application to sonic boom extrapolation. NASA Technical Paper. Langley Research Center, Virginia. Vol. 2214.

7. Kandil, O. A., Z. Yang, and P. J. Bobbitt. 2002. Prediction of sonic boom signature using Euler-full-potential CFD with grid adaptation and shock fitting. AIAA Aeroacoustics Conference.

8. Alauzet, F., and A. Loseille. 2010. High-order sonic boom prediction by utilizing mesh adaptive methods. AIAA Paper No. 2010-1390. 
9. Page, J. A., and K. J. Plotkin. 1991. An efficient method for incorporating Computational Fluid Dynamics into sonic boom prediction. AIAA Paper No. 91-3275.

10. George, A. 1968. Reduction of sonic boom by azimuthal redistribution of overpressure. AIAA Paper No. 68-159.

11. Salah El Din, I. 2004. Contribution à l'optimisation de la forme aérodynamique d'un avion de transport supersonique en vue de la réduction du bang. Thèse de doctorat. Université de Poitiers.

12. Rallabhandi, S., and D. Mavris. 2007. New computational procedure for incorporating computational fluid dynamics into sonic boom prediction. J. Aircraft 44(6).

13. Carrier, G., R. Grenon, V.-C. Le Pape, and I. Salah El Din. 2009. Sonic boom prediction methodology in use at ONERA and its application to sensitivity analysis of a supersonic business jet configuration. Canadian Aeronautics and Space Institute AERO'09 Conference. Aerodynamics Symposium.

14. Cambier, L., and M. Gazaix. 2002. elsA: An efficient object-oriented solution to CFD complexity. AIAA Paper No. 02-0108.

15. Taylor, A. D. 1980. The TRAPS sonic boom program. NOAA Technical memorandum ERL-ARL-87.

16. http://www.hisacproject.com/. 